\section{NIH Guidelines April 2019}

\author{
Esmeralda L. Meyer', Chris Jenkins ${ }^{2}$, and Kalpana Rengarajan'
}

Applied Biosafety:

Journal of ABSA International 2019, Vol. 24(4) 179-181

(C) ABSA International 2019

Article reuse guidelines:

sagepub.com/journals-permissions DOI: $10.1177 / 1535676019871146$ journals.sagepub.com/home/apb

@SAGE

\begin{abstract}
Following the required review period initiated in August 2018, the Department of Health and Human Services, National Institutes of Health (NIH), published the final changes to the Guidelines for Research Involving Recombinant or Synthetic Nucleic Acid Molecules (NIH Guidelines) in April 2019. This amendment focused on the review, registration, and reporting requirements for human gene transfer studies. In addition, the Recombinant DNA Advisory Committee (RAC) was renamed the Novel and Exceptional Technology and Research Advisory Committee (NeXTRAC) to serve as an advisory body on emerging technologies.
\end{abstract}

\title{
Keywords
}

$\mathrm{NIH}$ guidelines, human gene transfer, IBC, recombinant or synthetic nucleic acids, NIH

Following the required review period initiated in August 2018, the Department of Health and Human Services, National Institutes of Health (NIH), published the final changes to the Guidelines for Research Involving Recombinant or Synthetic Nucleic Acid Molecules (NIH Guidelines) in April 2019. ${ }^{1,2}$

Salient changes to the NIH Guidelines include the following:

1. The requirement to register and report human gene transfer (HGT) protocols to the NIH Office of Science Policy (OSP) was eliminated.

2. The Recombinant DNA Advisory Committee (RAC) was renamed the Novel and Exceptional Technology and Research Advisory Committee (NExTRAC) to align with the role initially assigned to the RAC as the advisory body on emerging biotechnologies.

3. Roles and responsibilities of investigators, institutional biosafety committees (IBCs), and the NIH were modified.

The changes to the NIH Guidelines are summarized in Table 1.

Relevant topics associated with the NIH Guidelines 2019 amendment are as follows:

1. Revised template for reporting incidents under the $\mathrm{NIH}$ Guidelines: https://osp.od.nih.gov/wp-content/uploads/ Incident-Reporting-Template_42319.docx

2. FAQs-April 2019 amendment of the NIH Guidelines: https://osp.od.nih.gov/biotechnology/faqs-on-the-nihguidelines-research-synthetic-nucleic-acid-molecules/
3. FAQs on IBC administration-May 2019: https://osp.od. nih.gov/biotechnology/faqs-on-ibc-administration/

4. FAQs on the vaccine exemption: Appendix M-III-A of the NIH Guidelines exempted certain vaccine trials from the NIH OSP registration requirements; these studies must comply with all other requirements of the NIH Guidelines: https://osp.od.nih.gov/wp-content/ uploads/2016/04/Vaccine \%20Exemption \%20FAQ\%20 sheet.pdf

Additional resources available to IBCs reviewing HGT studies are the following:

- IBCs may seek expertise outside the committee members and outside the institution.

- The points to consider included in the former Appendix $\mathrm{M}$ are now available on the NIH OSP web page "NIH Guidelines Frequently Asked Questions"

- RAC archives are available in the NIH OSP website.

- The ClinicalTrials.gov website contains information on publicly and privately supported clinical studies.

The Genetic Modification Clinical Research Information System $\left(\right.$ GeMCRIS $^{\circledR}$ ) will not be available after September 30, 2019. This database has not been updated since August 2018.

\footnotetext{
${ }^{\prime}$ Emory University, Environmental Health and Safety Office, Atlanta, GA, USA

${ }^{2}$ Clinical Biosafety Services, Wildwood, MO, USA

Corresponding Author:

Esmeralda L. Meyer, Emory University, Environmental Health and Safety Office, 1762 Clifton Road, Suite I200, Atlanta, GA 30322, USA.

Email: Evargas@emory.edu
} 
Table I. Comparison between the 2016 and 2019 Amendments to the NIH Guidelines.

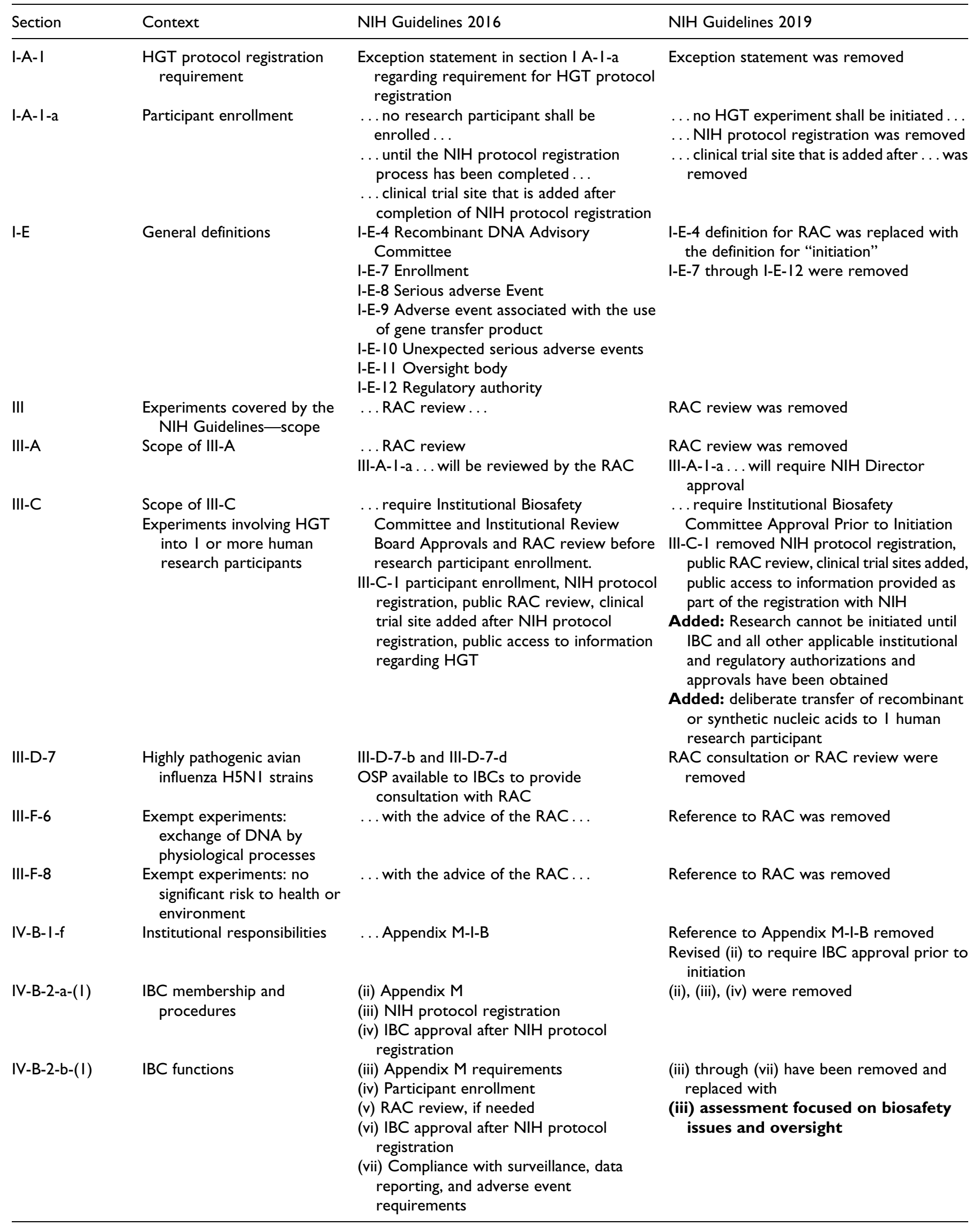


Table I. (continued)

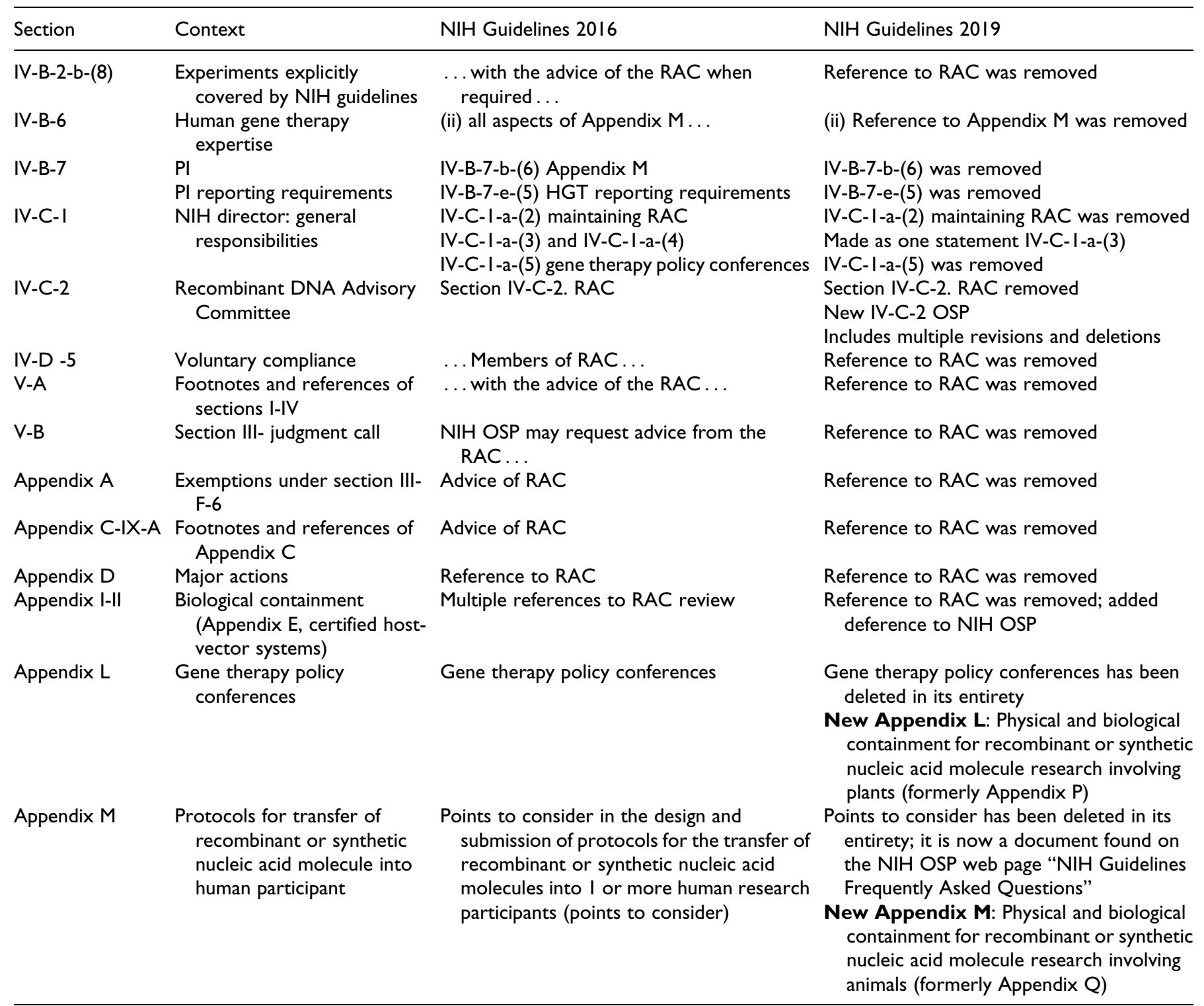

Abbreviations: HGT, human gene transfer; IBC, institutional biosafety committee; NIH, National Institutes of Health; OSP, Office of Science Policy; PI, principal investigator; RAC, Recombinant DNA Advisory Committee.

Inquiries can be directed to the NIH OSP at SciencePolicy@ od.nih.gov.

\section{Declaration of Conflicting Interests}

The authors declared no potential conflicts of interest with respect to the research, authorship, and/or publication of this article.

\section{References}

1. Federal Register Department of Health and Human Services Office of Science Policy National Institutes of Health. Final action under the NIH guidelines for research involving recombinant or synthetic nucleic acid molecules (NIH Guidelines). 84 FR 17858. https:// www.federalregister.gov/documents/2019/04/26/2019-08462/ final-action-under-the-nih-guidelines-for-research-involvingrecombinant-or-synthetic-nucleic-acid April 26, 2019. Accessed June 9, 2019.

2. Federal Register Department of Health and Human Services National Institutes of Health Office of Science Policy. Notice Proposed Changes to the Guidelines for Research Involving Recombinant or Synthetic Nucleic Acid Molecules. https://www. govinfo.gov/content/pkg/FR-2018-08-17/pdf/2018-17760.pdf August 17, 2018. Accessed June 9, 2019. 